\title{
MODELLING FREE RESPONSE OF A SOLAR PLANT FOR PREDICTIVE CONTROL ${ }^{*}$
}

\author{
Manuel Berenguel, Manuel R. Arahal and Eduardo F. Camacho
}

Departamento de Ingeniería de Sistemas y Automática, Universidad de Sevilla, Escuela Superior de Ingenieros, Avda. Reina Mercedes s/n, 41012 Sevilla, Spain

\begin{abstract}
This paper deals with the identification of a nonlinear plant by means of a neural network (NN) modelling approximation. The problem of neural identification is tackled using a static NN in a NARX configuration. A method is proposed to obtain the number of past values needed to feed the network. The on-line adaptation of the model and other issues are discussed. In order to show the benefits that can be achieved with the proposed methods, the NN model is used within a Model Predictive Control (MPC) framework. The MPC scheme uses the prediction of the output of the system calculated as the sum of the free response (obtained using the nonlinear $\mathrm{NN}$ model) and the forced response (obtained linearizing around the current operating point) to optimize a performance index. The control scheme has been applied and tested in a solar power plant.
\end{abstract}

Keywords: non-linear identification, neural networks, solar power plant.

\section{INTRODUCTION}

A key feature of the MPC schema is the necessity of a reliable model of the plant being controlled. The methodology of MPC for linear plants has to be changed when coping with nonlinear behaviour, because the superposition principle does not hold. Two aspects have to be revised: the model used for the output prediction and the optimization procedure to obtain the desired control signal.

Model for nonlinear output prediction- The nonlinear behaviour of the plant can be taken into account by using a nonlinear model. Neural network approaches constitute an adequate way to

- Work supported in part by CICYT Contract \#TAP95-0370 and IBERDROLA (Program "Ayudas a la Investigación Científica y al Desarrollo Tecnológico" - Proyecto 036/96). The experiments described in this paper were performed within the project "Enhancement and Development of Industrial Applications of Solar Energy Technologies", supported by EEC Program "Human Capital and Mobility - Large Installations Program" and promoted by CIEMAT - Plataforma Solar de Almería, Spain. obtain such kind of models. They have been applied to the identification of dynamical systems (Narendra and Parthasarathy, 1990) and used in a variety of model based controllers. When using static neural networks to predict future outputs of a system based on input-output information a problem is to determine the number of past values that the network needs to construct the prediction. A new selection method is proposed in this paper.

Off-line identification allows to develop and test a model before using it. It has the disadvantage that a large amount of data has to be stored. Selection of training examples among the available data has the benefits of reducing the training time and improving generalization. A simple procedure to select examples is commented in the paper.

After an off-line training phase, the network needs further on-line training. Fast on-line adaptation is achieved adjusting only a sub-network that faces small discrepancies and temporal changes. 
Optimization procedure- When nonlinear models are used within a MPC framework, computationally demanding and generally nonconvex nonlinear programs (NLP) arise (Camacho and Bordóns, 1995). Some algorithms have appeared in the literature to obtain less computationally demanding algorithms (de Oliveira and Morari, 1994). In this paper, the approach (proposed by authors in (Camacho and Berenguel, 1994)) used is based on a generalized predictive control (GPC) strategy. When coping with linear systems, a natural division of the system response in free and forced response can be obtained. The free response is interpreted here as the output obtained if the system input is maintained at a constant value during certain control and prediction horizons. If the deviation from linearity is not too large, some approximations can be made, which acknowledge that certain systems characteristics change from operating point to operating point, but assuming linearity in the neighborhood of a specific operating point. The algorithm used in this paper relies on the allowance of an adequate nonlinear model of the plant, obtained using static neural networks.

The paper is organized as follows: in section 2 a the neural identification problem is presented along with the proposed algorithm for selecting the inputs. Section 3 is devoted to describe the solar plant. In section 4 the controller structure which uses the neural model is defined. Finally, some issues regarding the application to the distributed collector field are discussed, including plant results.

\section{NEURAL IDENTIFICATION}

The problem we are interested in is the identification of nonlinear systems of the form:

$$
\begin{aligned}
y p_{(k+1)}= & h\left(y p_{(k)}, \cdots y p_{(k-L)},\right. \\
& u_{(k)}, \cdots u_{(k-M)}, \\
& \left.p_{(k)}, \cdots p_{(k-N)}\right)
\end{aligned}
$$

where $u_{(k)}$ is the system's input vector at time $k$, $y p_{(k)}$ is the output vector, $p_{(k)}$ is a vector of measurable disturbances and $h$ is an unknown nonlinear function.

For the identification of plant (1), a neural network model like the one depicted in Fig. 1 can be used. Tapped Delay Lines (TDL) provide past values of the variables. This structure is called NARX model. The prediction is given by:

$$
\begin{aligned}
\hat{y}(k+1)= & f\left(y p_{(k)}, \cdots y p_{(k-P)},\right. \\
& u_{(k)}, \cdots u_{(k-Q)}, \\
& \left.p_{(k)}, \cdots p_{(k-R)}, W_{f}\right)
\end{aligned}
$$

where $W_{f}$ is a vector containing all the parameters in the neural network.

Two problems have to be solved. First of all, a model has to be selected among the family of NARX models. This is the model selection problem and includes selecting the number of nodes, connections, etc. of the NN. A second step is to give a value to the adjustable parameters of the model. This is called the parameter estimation problem. The model selection problem is tackled here in two steps: first the inputs that feed the network are selected, later, the neural structure is determined.

\subsection{Selection of past values as network inputs.}

The temporal domain is considered treating past values of variables as different inputs and feeding them to a static network. Taking an upper bound of the order (when it is unknown) can lead to inefficient models due to the large number of inputs needed. A new method is proposed, based on the descent in the gradient needed to explain the observed output when a new TDL is added. The method chooses the variable that provides the largest descent at each stage, determining $P, Q$ and $R$ in (2) so as to obtain a good approximation to the "supposed true" plant (1). For real processes, the larger $P, Q$ and $R$, the more accurate the approximation can be made. It is clear, however, that an arbitrary large number of inputs can turn our problem insolvable. Furthermore, in order to avoid over-parametrization it is convenient to keep the number of adjustable parameters as small as possible.

To expose the idea that supports the method, let us assume that the components of vector $\mathrm{x}$

$$
\begin{gathered}
\mathbf{x}_{L M N(k)}=\left[y p_{(k)}, \cdots y p_{(k-L)},\right. \\
u_{(k)}, \cdots u_{(k-M)}, \\
\left.p_{(k)}, \cdots p_{(k-N)}\right]
\end{gathered}
$$

suffice to construct the prediction as $\hat{y}(k+1)=$ $f\left(\mathbf{x}_{L M N(k)}\right)$. If one tries to approximate function $f$ using an input vector $\mathbf{x}_{P Q R}$, with dimension less than $\operatorname{dim}\left(\mathbf{x}_{L, M, N}\right)$, then it is likely to find a couple of values for $\mathrm{x}_{L, M, N}$ that produces the same value for $\mathrm{x}_{P Q R}$. In this case, the model based on $\mathrm{x}_{P Q R}$ will yield the same prediction and the real plant will give different outputs. From the point of view of the model, the output of the system is multivalued. The number of past values of $u, y p$ and $p$ can be increased until the ambiguity in the output of the model disappears. For a couple of instancies of vector $\mathbf{x}_{P Q R}$, such as $\xi^{1}, \xi^{2}$ the gradient of function $f$ along the line that joins both points can be estimated as $\left|\nabla_{12} f\right| \approx \frac{\left|v^{1}-v^{2}\right|}{\| \xi^{1}-\xi^{2}||}$, provided that the distance $\left\|\xi^{1}-\xi^{2}\right\|$ is small enough and 

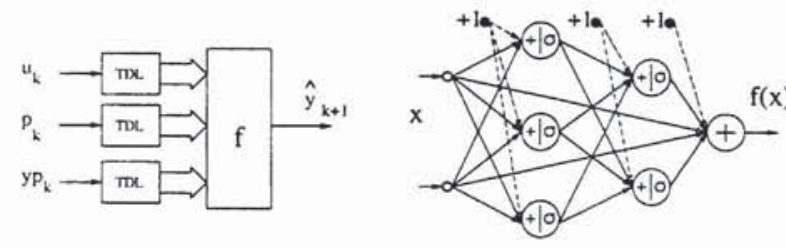

Fig. 1. NARX model (left) and MLP with 2 hidden layers (right).

being $v^{i}$ the correct value for function $f$ at points $\xi^{i}$ with $i=1,2$.

It is easy to see that, for a given $\xi^{1}, \xi^{2}$ pair, the value of $\left|\nabla_{12} f\right|$ can be decreased augmenting both vectors with some new past value that explains the difference in the values of the output. With this idea in mind, the algorithm can be expressed as follows: $\square$ Gather a set of input-output data from the plant. This set should be informative enough of the dynamical behaviour of the plant in most relevant operating points.

$\square$ Begin with small values for $P, Q$ and $R$.

$\square$ Create a set of exemplars consisting of pairs in the form $\mathbf{z}:=\left(\mathbf{x}_{P Q R}, v\right)$, where $\mathbf{x}_{P Q R}$ is the model's input vector defined in (2), and $v$ is the correct output for said input. Let us call this set $A_{P Q R}$.

$\square$ For each pair $\mathbf{z}^{1}, \mathbf{z}^{2}$ in $A_{P Q R}$.

1. Compute $d_{z}=\left\|\mathrm{z}^{1}-\mathrm{z}^{2}\right\|$.

2. If $d_{z}<\Delta$ estimate the gradient. Store the $z^{1}$, $\mathrm{z}^{2}$ pair and the value calculated for the gradient in a separate set $B_{P Q R}$.

$\square$ Increase $P, Q$ and $R$ in one unit separately and choose the combination that produces the largest descent in the gradient for each pair in $B_{P Q R}$.

$\square$ If the criteria for stopping is met (see discussion below), then finish, otherwise create a new set of exemplars with the new values of $P, Q$ and $R$ and repeat the procedure.

The parameter $\Delta$ is the largest distance between pairs in the input space to be considered close enough to compute the gradient. If all signals are normalized to the $[-1,1]$ interval, then $\Delta$ can be taken as $\Delta=\sqrt{\operatorname{dim}(\mathbf{z}) \delta^{2}}$, where $\delta$ defines a grid in the input space that can be viewed as a fraction of the whole scale for the normalized variables.

The algorithm can be stopped according to some criteria. One could be when a maximum prespecified number of inputs is reached. Another is when the maximum value of the gradients is below some threshold. The algorithm can be run a number of times and develop the model for each selection, taking finally the one that gives the best complexity/performance trade-off.

\subsection{Neural structure selection.}

In this paper, a 2-hidden-layer net is used as the one shown in Fig. 1. The nonlinear activation is given by the function $\sigma(s)=\frac{1-e^{-s}}{1+e^{-s}}$. Bias weights are set up as connections to nodes with constant values, as shown in Fig. 1. Direct connections from the input to the output layer provide a means to approximate linear mappings easily. This structure is capable of approximating any continuous non-linear mapping provided that a sufficiently large number of nodes is used. The number of nodes is determined training different networks and determining the approximation and generalization capabilities using cross-validation. If the results are not good enough, the number of nodes is increased and the procedure repeated.

\subsection{Selection of training set.}

Training a neural network to approximate an unknown input-output relation from examples typically involves a large number of cycles through the set of available examples. For this reason it is convenient to have a small training set (TS) with the higher information content. When the inputoutput patterns or exemplars come from a system under control the variables are highly correlated to each others and the information content is low. The use of some technique to select exemplars to be included in the TS has the beneficial side-effect of improving generalization removing redundant patterns.

For the purpose of training a neural network to identify the solar plant, a procedure has been devised to extract a compact yet informative enough data set. The main idea behind the method is that one should include in the TS only inputoutput patterns whose distance to other patterns already in the set is large enough. The distance can be taken as the Euclidean distance in the input-output space, but, since not all signals affect the output the same extent, it is more convenient to use some weighted metric, defined as $\|\mathbf{v}\|_{w}^{2}=\mathbf{v}^{T} \mathbf{W}^{T} \mathbf{W} \mathbf{v}$, with $\mathbf{v}=\mathbf{z}_{1}-\mathbf{z}_{2}$, being $\mathbf{z}=(\mathbf{x}, v)$ a point in the input-output space. The network's input is $\mathbf{x}$ and $v$ is the correct prediction for such input. Matrix $\mathbf{W}$ allows the more relevant components to be better taken into account using a priori knowledge about the plant.

The method followed can be summarized as follows:

$\square$ Gather all available input-output data in a set of candidate examples (CE set).

$\square$ Take the first instance $\mathbf{z}$ in the CE set and add it to the set of selected patterns (SP set).

$\square$ For each $\mathbf{z}$ in the CE set.

1. Compute $d:=\Upsilon(\mathbf{z}, S P):=\min _{\theta \in S P}\|z-\theta\|_{w}$, minimum distance from $\mathbf{z}$ to an element of SP.

2. If $d>d_{\min }$ include $\mathbf{z}$ in SP. 


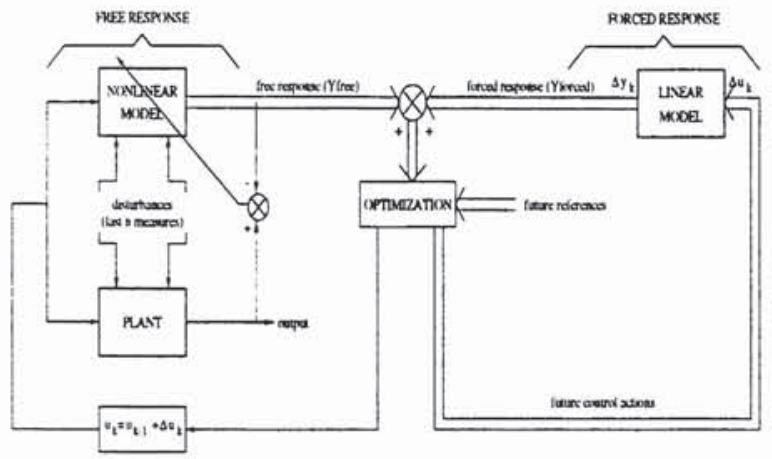

Fig. 2. Nonlinear MPC scheme using a linear model for the forced response and a neural network for the free response.

This method is simple to implement and gives good results. The only parameter one has to choose is the minimum distance between patterns in the SP set. This value can be approximated by trial and error beginning with a high value and reducing it using cross-validation to check generalization.

\section{BRIEF DESCRIPTION OF THE PLANT}

The ACUREX distributed collector field facility is at the Plataforma Solar de Almería (Spain). It consists of a series of parabolic mirrors that reflect solar radiation onto a pipe where oil gets heated while circulating. A pump extracts cold oil from the bottom of an storage tank injecting it on the field. At the output of the collector field the oil enters the upper part of the storage tank.

The objective of the control system is to maintain the outlet oil temperature at a desired level in spite of disturbances such as changes in the solar irradiance level (caused by clouds), mirrors reflectivity or inlet oil temperature changes. The only manipulable variable is the flow of oil.

\subsection{Plant dynamics due to input signal.}

Step-response and PRBS (Pseudo Random Binary Sequence) tests were carried out at the plant in an open loop configuration to obtain low order linear models of the field for control purposes. The plant exhibits a number of antiresonance modes (frequencies at which the magnitude of the frequency response changes strongly). High order linear models have been developed to take into account these modes (Camacho et al., 1994). For control purposes, the distributed collector field can be approximated by a linear system when considering small disturbances. This is a good approximation if only low frequencies are excited. If this is not the case, as happens when more de- mands are made in the plant response time, the anti-resonance modes (unmodeled dynamics) may originate an unacceptable oscillatory behaviour.

\section{APPLICATION TO THE SOLAR PLANT}

In the standard formulation of GPC, linear CARIMA models can be used to model the plant, yielding a linear optimization problem (Camacho and Bordóns 1995). The optimal GPC control actions are calculated by computing a vector $\mathbf{u}$ of future control increments that minimizes the objective function.

In order to solve the optimization problem for nonlinear plants, the control structure presented in Fig. 2 can be used. The free response is calculated by using a nonlinear prediction model of the system and the forced response using a linearization model around the operating point. This scheme allows to calculate the incremental control signal $\mathbf{u}$ in the standard way, without the need for nonlinear optimizations.

Several models have to be developed for the prediction of the free and forced response of the system subject to different disturbances. Some models use physical knowledge about the processes and do not need but some parameter estimation.

The free response of the plant is due to little known non-linear processes and black box modelling is of use. The algorithms exposed in previous sections have been applied using input-output data previously obtained from the plant. For the purpose of training a neural network to identify the plant, the procedure mentioned in section 2 was devised to extract a compact yet informative enough data set. Using this data set, the neural structure, the number of past inputs, and the network adjustable parameters were selected. In the following, the development of the different submodels is exposed.

\subsection{Neural structure selection.}

For the identification of the solar plant, a small network of 10 input nodes, 6 nodes in the first hidden layer, 5 nodes in the second hidden layer and a linear output node gives good results. This was found using a procedure that increases the network size, trial after trial until the performance does not increase significantly.

The procedure was carried out using two disjoint sets: one for training and the other for checking the results after some epochs. The results obtained for some of the networks tested are: 


\begin{tabular}{ccc}
\hline network & $E_{r m s}(\mathrm{TS})$ & $E_{r m s}(\mathrm{VS})$ \\
\hline $10-4-4-1$ & 0.016 & 0.024 \\
$10-5-4-1$ & 0.015 & 0.020 \\
$10-5-5-1$ & 0.012 & 0.019 \\
$10-6-5-1$ & $\mathbf{0 . 0 0 9}$ & $\mathbf{0 . 0 1 8}$ \\
$10-7-6-1$ & 0.008 & 0.018 \\
\hline
\end{tabular}

the second and third columns indicate the final root-mean-squared error in the training set (TS) and in the validation set (VS). The network structure is denoted in the first column by the number of nodes in each layer. The values in said table correspond to an average over several runs since the initial random state of the net can influence the result. The number of cycles is different for each run, since training was stopped to avoid overtraining.

\subsection{Selection of inputs.}

For the prediction of the free response, the variables of interest are:

$\square$ Input variable: oil flow. The number of past values is denoted by $P$.

$\square$ Output variable: output temperature. The number of past values is denoted by $Q$.

$\square$ Perturbations: inlet temperature and solar radiation. The number of past values are denoted by $R_{1}$ and $R_{2}$, respectively.

In Fig. 3 the procedure is exemplified. Each box in said figure represents a different $B_{P Q R 1 R 2}$ set. It is clear that the proposed algorithm avoids the exploring of the whole tree. The value of $\delta$ was taken as a one percent of the full-scale range for the normalized variables, that is $\delta=0.01$. The results obtained with the proposed algorithm for the first stage are:

\begin{tabular}{ccccccc}
\hline$P$ & $Q$ & $R_{1}$ & $R_{2}$ & $\vec{r}$ & $e_{r m s}$ TS & $e_{r m s}$ VS \\
\hline 2 & 1 & 1 & 1 & $79 \%$ & 0.0112 & 0.0140 \\
1 & 2 & 1 & 1 & $82 \%$ & 0.0115 & 0.0150 \\
1 & 1 & 2 & 1 & $\mathbf{8 9} \%$ & $\mathbf{0 . 0 0 5 4}$ & $\mathbf{0 . 0 0 7 7}$ \\
1 & 1 & 1 & 2 & $86 \%$ & 0.0115 & 0.0150 \\
\hline
\end{tabular}

the 5th column shows the averaged gradient reduction. These results where tested training all intermediate models, resulting in the approximation errors given in columns 6 (TS) and 7 (VS).

\subsection{Selection of the training set.}

The application of the algorithm to the selection of a TS for the identification of the solar power plant gave very good results. The method provided a TS with just $30 \%$ of the total number of examples, reducing thus the training time. Moreover, the generalization capacity of the resulting network was tested after training and compared

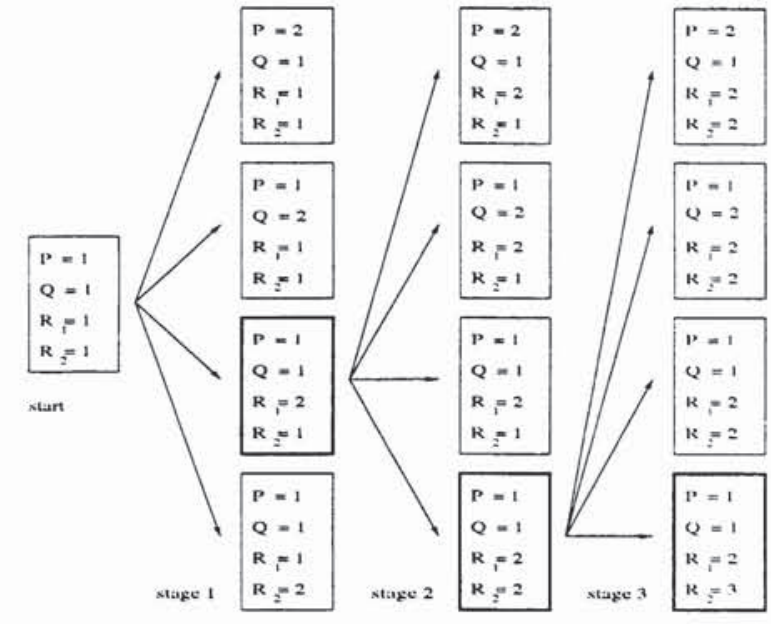

Fig. 3. The algorithm for the selection of inputs in its 3 first stages.

with another network that was trained using the whole data set. The comparison revealed that the pruning of redundant examples lead to a better generalization.

\subsection{On-line adaptation.}

On-line adaptation is desirable to counter temporal changes and model mismatch due to incomplete learning. Adapting a neural net with a large number of connections needs a lot of computing time. In order to achieve on-line adaptation with just a few adaptable parameters, a small subnet was added to the previously trained one. In the forward path of backpropagation (BP), both nets provide an output that are added at the output node. In the feedback pass of BP only the small subnet is adapted. The structure of the subnet is fixed and selected ad hoc, having 10 input nodes, 4 hidden nodes and sharing the output node with the non adaptable network.

\subsection{Disturbance prediction models.}

Some studies have been performed (Camacho et al., 1992) to relate changes in outlet oil temperature to inlet oil temperature variations, but the benefits provided in this control scheme were not considered to justify the added complexity and so, the inlet temperature has been considered constant along the prediction horizon in the optimization phase of the algorithm.

Solar radiation changes due to its daily cycle and to passing clouds. The prediction of passing clouds is very complex and so, an approximation based on the consideration of a clear-day operation has been adopted (Berenguel, 1996). The predicted values are used in the neural network nonlinear model to calculate the free response. 


\section{CONCLUSIONS}

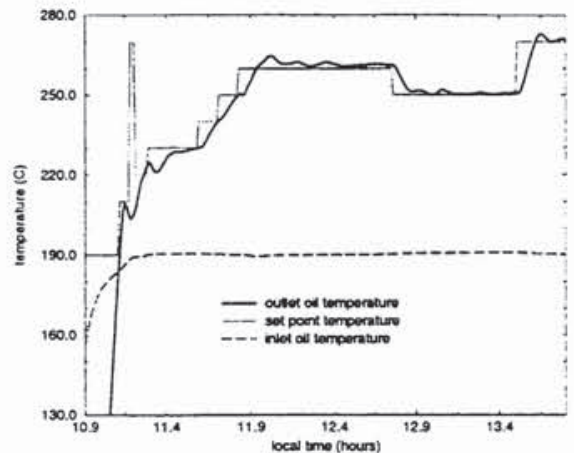

set point, inlet and outlet oil temperatures $\left({ }^{\circ} \mathrm{C}\right)$.

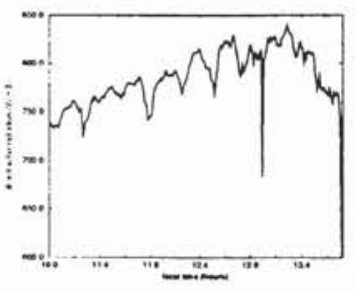

solar radiation $\left(\mathrm{W} / \mathrm{m}^{2}\right)$.

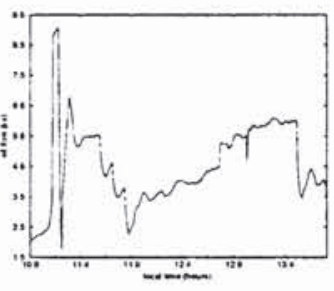

oil flow $(1 / s)$
Fig. 4. Test with the neural nonlinear GPC controller $(03 / 07 / 96)$.

\subsection{Forced response calculation.}

If set point changes are small (less than $20^{\circ} \mathrm{C}$ ), the hypothesis of operation around an operating point in the forced response calculation is a right one and linear models in usual CARIMA formulations can be used. The CARIMA models are obtained from the PRBS identification data for operation about medium flows. Theoretically, this model should change for different operating points. In this particular case, due to the large influence of solar radiation in the value of the control signal, the contribution of the linear part in the control signal is smaller than that of the free response.

\subsection{Plant results.}

The MPC control scheme has been applied to the control of the distributed solar collector field ACUREX.

Fig 4 shows the results obtained in a day with scattered and passing clouds which produce changes in solar radiation that disturbed the outlet oil temperature level during the operation. The behaviour of the controlled system is very acceptable, with good set point tracking and disturbance rejection characteristics. The results obtained with this controller have been compared with other control approaches previously tested at the plant, showing very good performance characteristics.
The identification of a complex non-linear system subject to disturbances have been carried out using neural networks. As a part of the process, the model selection problem has been tackled using the NARX structure and a simple algorithm to determine the number of TDLs needed for it. The model has been used in a model-based predictive control scheme. The control algorithm uses the neural network model to predict the free response of the plant and a linear incremental model to compute the optimal control action.

Aspects of the neural identification such as the selection of training samples and the realization of fast, on-line adaptation have been commented. Finally, results have been presented showing the control performance in the real plant.

\section{ACKNOWLEDGMENTS}

The authors would like to thank personnel of the Solar Platform of Almería, especially P. Balsa.

\section{REFERENCES}

Berenguel, M. (1996). Contribuciones al Control de Colectores Solares Distribuidos. $\mathrm{PhD}$ thesis. Universidad de Sevilla.

Camacho, E.F. and C. Bordóns (1995). Model Predictive Control in the Process Industry. Springer-Verlag.

Camacho, E.F. and M. Berenguel (1994). Application of Generalized Predictive Control to a Solar Power Plant. In: Proc. of The Third IEEE Conference on Control Applications, Glasgow, UK. pp. 1657-1662.

Camacho, E.F., F.R. Rubio and F.M. Hughes (1992). Self-tuning Control of a Solar Power Plant with a Distributed Collector Field. IEEE Control Systems Magazine pp. 72-78.

Camacho, E.F., M. Berenguel and F.R. Rubio (1994). Application of a Gain Scheduling Generalized Predictive Controller to a Solar Power Plant. Control Engineering Practice 2(2), 227-238.

de Oliveira, S. and M. Morari (1994). Robustly stabilizing receding horizon control for constrained nonlinear systems. In: Proc. American Control Conf., Baltimore, MD.

Narendra, K. S. and K. Parthasarathy (1990). Identification and control of dynamical systems using neural networks. IEEE Transactions on Neural Networks 1, 4-27. 